$\mathrm{DE}$

M E D I C I N A

T R O P I C A L

$\mathrm{DE}$

SÃO PAULO

JOURNAL OF THE SÃO PAULO INSTITUTE OF TROPICAL MEDICINE

(1)Universidad Científica del Sur, Laboratorio de Biología Celular, Lima, Peru

(2)Universidad Nacional Mayor de San Marcos, Escuela de Biología, Laboratorio de Microbiología, Lima, Peru

${ }^{(3)}$ Hospital Nacional Guillermo Almenara Irigoyen, Departamento de Microbiología, Lima, Peru

${ }^{(4)}$ Hospital Nacional Guillermo Almenara Irigoyen, Departamento de Neurología, Lima, Peru

(5)Tulane University, School of Public Health and Tropical Medicine, New Orleans, United States

Correspondence to: Jesús Rojas-Jaimes Universidad Científica del Sur, Km 19, Villa, Panamericana Sur, Villa EL Salvador, 01511, Lima, Peru

Tel: +511993638840

E-mail: jesus.rojas.jaimes@gmail.com

Received: 18 May 2017

Accepted: 5 September 2017

\section{Subdural empyema caused by Peptostreptococcus sp.: a complication of acute pharyngitis}

\author{
Jesús Rojas-Jaimes ${ }^{1,2}$, Alberto Diaz-Tello ${ }^{3}$, Cristian Carpio Bazán ${ }^{4}$, Meredith \\ Kerrigan ${ }^{5}$
}

\section{ABSTRACT}

Subdural Empyema (ESD) is the collection of purulent fluid that develops between the exterior "dura mater" layer and the middle "arachnoid mater" layer that covers the brain. ESD can be caused by a primary infection located in the paranasal sinuses. In many aerobic and/or anaerobic bacterial cases, hearing or traumatic processes serve as the causative agent. This report presents pharyngitis in a young girl which later developed into a subdural empyema caused by the bacteria Peptostreptococcus sp. The report emphasizes the correct clinical valuation of pharyngitis as a risk factor for developing subdural empyema in children.

KEYWORDS: Subdural empyema. Peptostreptococcus sp. Collection infection. Pharyngitis.

\section{INTRODUCTION}

A Subdural Empyema (ESD) is defined as a collection of purulent fluid in the space situated between the internal level of the dura mater and the external membrane of the arachnoid mater. It is a rare clinical condition,accompanied by a higher morbidity and mortality if not diagnosed and treated early ${ }^{1}$.

In past decades, Haemophilus influenzae has been found to be the primary cause of intracranial infections corresponding to $25 \%$ of ESD cases. Currently, the vaccine has drastically reduced the number of cases $^{1,2}$. The ESD risk process allows the infection to disseminate in the cerebral hemispheres leading to the subsequent development of an encephalic abscess ${ }^{1}$.

Peptostreptococcus sp. is an aggressive non-spore forming cocci bacteria. It is a gram-positive, anaerobic bacteria which is linked to bone, ear, eye, joints, deep neck areas and cerebral infections, serving in some cases as a cryptogenic site (without an obvious focus of initial infection), presuming a migration of the upper respiratory tract and/or microbiota from the mouth to cerebral regions ${ }^{3-5}$; this can be observed in the development of abscesses during surgery recovery ${ }^{6,7}$. Abscesses in the central nervous system are a challenge for neurosurgeons, because of the delicate draining procedure, and for microbiologists that must identify the causative agent that, in many cases, is underdiagnosed due to contamination of the sampling procedure and/or difficulties in the cultivation of the purulent fluid especially in anaerobic conditions ${ }^{3}$. A 24-year retrospective study with 352 cerebral abscesses was performed. Patients were in general male, younger than 2 years of age or over 80 years of age suffering from other underlying conditions, specifically in the frontal and parietal areas of the brain $^{3}$. Cases of acute meningitis caused by anaerobic bacteria are less than $1 \%$ with the majority caused by Peptostreptococcus magnus. In certain cases, there was a synergy of various infections, occurring in episodes that resemble sinusitis and pharyngitis ${ }^{6-9}$. 
Peptostreptococcus magnus is often the causative agent of respiratory tract infections such as sinusitis and otitis media (middle ear), infections of the abdomen, of female genitourinary tract, mouth (gums and teeth), and central nervous system. The latter location becomes infected because of the bacterial migration from primary foci of infection. As previously described in many cases, it can lead to a fatal infection at this stage ${ }^{3,10,11}$. P. magnus can be found in the oral cavity and can colonize and cause infections when the epithelial barrier is damaged by the physiopathological process, prior to surgery ${ }^{10,12,13}$.

The medical protocol for treating an infection caused by Peptostreptococcus sp. is a neurosurgical intervention to release the intracranial pressure while prescribing antibiotics like metronidazole that spread well throughout the central nervous system, cephalosporins and carbapenems ${ }^{6}$.

The present study aimed at calling attention to acute pharyngitis as a risk factor for subdural empyema caused by Peptostreptococcus sp. in a patient without significant comorbidities.

\section{CASE REPORT}

A 12-year-old female patient with no previous referred diseases complained of a one-week persistent cephalalgia that was diagnosed at a hospital in Cerro de Pasco, Peru. At the time the patient had fever and acute pharyngitis. She received metamizole and paracetamol. Five days later high fever and a major headache persisted, and were aggravated by nausea, vomiting, four days of paresthesia and weakness in the inferior left limb. A computed axial encephalic tomography indicated a subdural hematoma in the right frontoparietal section, and the patient was immediately transferred to the emergency room at the National Guillermo Almenara Irigoyen Hospital. A neurological evaluation, as well as an electrocardiogram were performed. Electrocardiogram (ECG) 13 (O3, V4, M6), in apparent good regular conditions (AREG); spontaneously breathing, right pupils at $4 \mathrm{~mm}$ and left at $3 \mathrm{~mm}$ and reactive, left hemiparesis $3 / 5$, drowsy, stiff neck $+/+++$, on the computed axial CT scan, "TAC" showed a subdural right frontoparietal fluid that contrasted with a slightly moderate mass effect (Figure 1). In the preoperative assessment of the patient, no cause of immunosuppression was diagnosed. Additionally, in the evaluation, we did not identify any calvarial defects or calvarial erosions by $\mathrm{CT}$. The conclusion was a subdural empyema caused by dural damage due to the hematological spread of infection.

In the laboratory exams (CSF cerebrospinal fluid): 90 cells (70\% MN, 30\% PMN); elevated glucose: 74 $\mathrm{mg} / \mathrm{dl}$ and protein: $48 \mathrm{mg} / \mathrm{dl}$ normal; coagglutinations

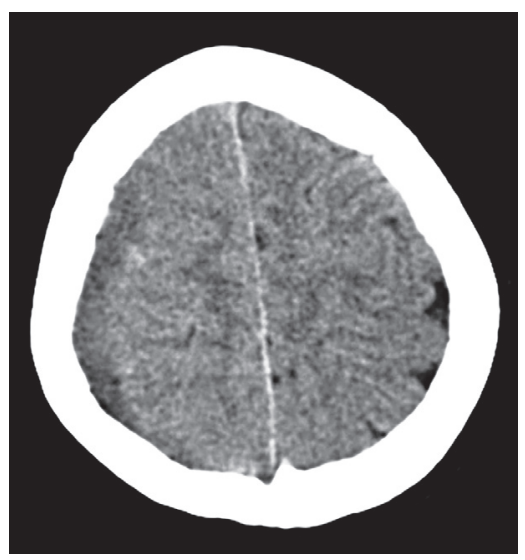

Figure 1 - Localization of fluid in the right subdural frontoparietal area observed in a computed tomography axial without contrast

for Neisseria meningitidis Group B/E and A and C, Haemophilus influenzae, Streptococcus pneumoniae, Group B Streptococcus all negative; complete blood count: leukocytes $18.600 / \mathrm{mm}^{3}$ with band neutrophils at 5\%; PCR: $347 \mathrm{mg} / \mathrm{dl}$. The subdural empyema diagnosis was based on a right frontoparietal decompressive craniectomy, and a stressed subdural empyema evaluation, continuous irrigation. The bone plate was deposited in the abdominal wall. The purulent fluid was sent to the department of microbiology in the hospital and cultivated in anaerobic agar and in a bottle for anaerobics and was incubated at $37^{\circ} \mathrm{C}$ for $24 \mathrm{~h}$ to allow the microbiologic classification of Peptostreptococcus sp. that was confirmed by Gram staining (Figure 2). The patient was then transferred to the Pediatric Intensive Care Unit where she received postoperative care and progressed favorably; the drainage was removed and evidence of recuperation reached a 15 point in the Glasgow scale without motor or sensory impairment. The patient was transferred to neurosurgery and received antibiotic therapy

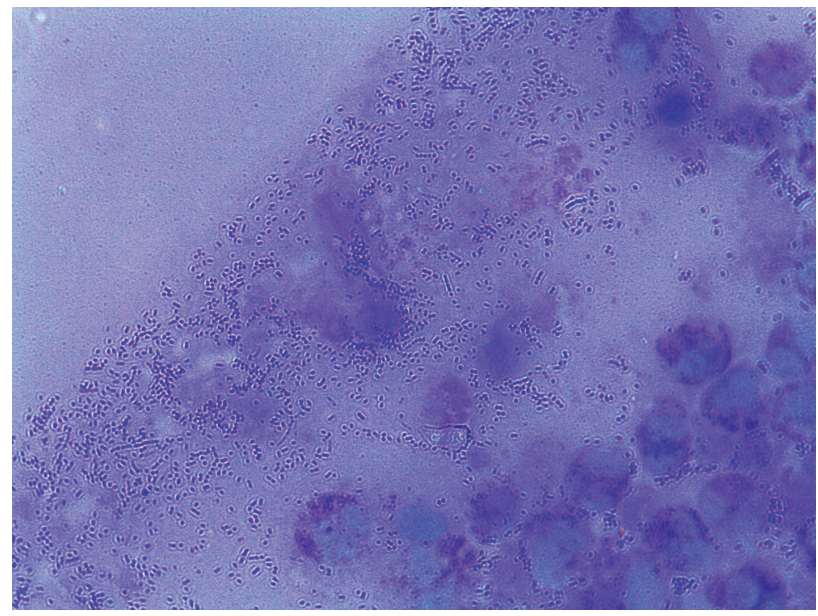

Figure 2 - Peptostreptococcus sp. and polymorphonuclear leukocytes from the purulent fluid observed by Gram staining at $1,000 \mathrm{X}$ magnification 
consisting of Meropenem, Vancomycin, and Metronidazole and was discharged after 17 days in the hospital. The reason for using a triplet antibiotic was based on the characteristics of the broad spectrum of antibiotics against the bacteria and the unknown etiological agent at the time of treatment. Additionally, continued outpatient antibiotherapy was maintained for two weeks post-discharge. Monitoring of encephalic healing with TAC was performed on the right frontoparietal encephalomalacia four months later. Seven months after hospital discharge the patient received cranioplasty with miniplates to restore the skullcap.

\section{DISCUSSION}

Although the use of contrast is ideal, the computed axial tomography (TAC) without contrast is a great instrument for diagnosing initial subdural empyema as observed in this study ${ }^{1}$.

On the sheet stained with Gram containing the purulent fluid that was obtained after craniectomy, one could observe typical Gram-positive cocci of Peptostreptococcus sp. with an abundant quantity of leukocytes, although, in some cases, the sheet observation appears negative probably due to the purulent nature of the sample ${ }^{6}$. In contrast, the culture is more sensitive and reported better results in the identification of the etiological agent compared to the sheet reading. In our study, we observed that culture for anaerobic bacteria could identify pure colonies of Peptostreptococcus sp. However, in some cases, the isolation of the agent is difficult due to increased susceptibility to contamination in anaerobic conditions, as well as possible contamination due to transport and extraction ${ }^{12}$. It is important to note that Peptostreptococcus sp. is undervalued as a causative agent of infections, as previously prescribed, and with a good protocol and improved diagnostics one could identify the real association of these bacteria with skin, bone and joint infections, endocarditis, necrotizing pneumonias and meningitis. In the latter case, Peptostreptococcus sp. has been reported as a causative agent of a progressive deadly infection ${ }^{14}$.

The great vascularization of the pharynx and the development of the localized infection at this stage of life could be explained as a risk of ESD caused by Peptostreptococcus sp., although ESD is related,in many cases, to a sinusitis predominantly in male children and adolescents, ESD also is associated with traumatic lesions and post-craniotomies. It is clinically important to mention that, in rare occasions, subdural lesions are associated with meningitis by which the intervention of the subdural level should be prompt and correct with surgical management and antibiotics ${ }^{1,15}$.
The most frequently occurring clinical symptoms of ESD in children and adolescents are fever, headache, irritability, nausea, vomiting and altered states of conscience. This symptomatology can last one to two weeks after the confirmation of diagnosis, therefore, the suspicion of ESD in a patient with fever and respiratory infection and later neurological symptoms is of medical importance ${ }^{1}$. These descriptive characteristics contrast with the progression of the illness in the reported case, which was diagnosed with pharyngitis as the only determining factor of infection and evolved with nausea, vomiting and paresthesia of the inferior left limb as aggregate important factors.

The infection route of ESD is determined by its etiology; for example, in the infections of paranasal sinuses, the frequently isolated agents are aerobic Strepcoccus and anaerobic Streptococcus intermedius. In addition, it is relevant to mention that it is very rare to isolate Streptococcus pneumoniae despite the bacteria being a frequent agent of sinusitis in children, even if studies report it as a frequent agent of meningitis ${ }^{1,16,17}$. In postoperative and posttraumatic infections, the most frequently isolated agents are Staphylococcus aureus and coagulase negative Staphylococcus ${ }^{1}$. It is important to emphasize that ESD caused by a pharyngitis is rare. A prior study showed ESD related to pharyngitis in a 7-year-old child with a 7-day fever, even though the etiologic agent was Streptococcus pyogenes $^{18}$. In contrast with our study, one can isolate Peptostreptococcus sp. as a causative agent of ESD.

Treatment of ESD is medical and surgical; medically, it consists of using broad-spectrum antibiotics, like third-generation cephalosporins, carbapenems and metronidazole ${ }^{18-20}$, more efficient to treat aerobic agents than aerobic ones, during three to four weeks post-surgical drainage. This regimen contrasts with the treatment that is established for the patient prescribed with meropenem, metronidazole and vancomycin, although the latter is recommended to treat ESD before surgeries or after trauma due to the presumption of microbial contamination ${ }^{1,6}$. Current disease guidelines mention that, in infants and young children, ESD could be the aftermath for lifetime complications, if not properly managed, so the imaging in the primary diagnosis as Computed Tomography CT or Cranial ultrasonography are recommended ${ }^{21}$. In our case, $\mathrm{CT}$ identified the ESD. The surgical treatment was craniectomy to allow the complete evacuation of empyema, as recommended in a previous study $^{21}$. Antibiotics management depends on the route of infection. Vancomycin, ceftriaxone plus metronidazole should be supplied when the etiological agent is unknown and drug resistance is probable ${ }^{21}$. At the time of antibiotics use, we did not know the etiological agent; therefore, we used three antibiotics to cover a range of bacteria that 
could have caused the infection in the patient; vancomycin (Gram-positive cocci), meropenem (Gram-negative bacilli) and metronidazole (anaerobic cocci). The recommended surgery is a decompressive craniectomy with the advantage of reaching the area of infection to remove the necrotic tissue and drain the purulent fluid, unlike a trepanation ${ }^{1}$. In the present case, the intervention was carried out by the decompression of the right frontoparietal region and, as recommended by previous studies, and the drainage of purulent fluid from the subdural empyema ${ }^{1}$.

It is recommended that a neurosurgeon and an otolaryngologist perform a continued postoperative evaluation to ensure the favorable progression of the patient or if the patient requires new surgical interventions, for instance to drain persisting or recurring fluids ${ }^{1}$. In our study, only one surgical intervention was carried out to correctly drain the purulent fluid.

Lethality of ESD varies between $6 \%$ and $17 \%$, becoming the posttraumatic cause associated with the highest mortality. Regarding morbidity, post-ESD is associated with persistent neurologic impairment, hemiparesis, and convulsions in $40 \%$ of cases ${ }^{1}$.

Study limitations were that pharyngitis was classified only by the patient's signs and symptoms and no sample was taken initially to identify the causative agent Moreover, in respect to the subdural empyema, classical microbiology was used only to identify the genus Peptostreptococcus sp., but not the identify the species.

It can be concluded that pharyngitis can be a risk factor for development of subdural empyema in children; therefore, it is important to correctly validate pharyngitis at an early stage and implement the follow-up and the clinical treatment without dismissing the case.

\section{REFERENCES}

1. Bustos RO, Pavéz PA, Bancalari BJ, Miranda RM, Escobar HR. Empiema subdural como complicación de sinusitis. Rev Chilena Infectol. 2006;23:73-6.

2. Kobayashi Y, Sunakawa K, Fujita K, Saijo M, Murono K, Sakata $\mathrm{H}$, et al. Trend of bacterial meningitis in children over a 14 year period (1981 through 1994) in Japan - an analysis based on studies in 27 institutions. Kansenshogaku Zasshi. 1997;71:1017-24.

3. Lakshmi V, Umabala P, Anuradha K, Padmaja K, Padmasree C, Rajesh a, et al. Microbiological spectrum of brain abscess at a tertiary care hospital in South India: 24-year data and review. Patholog Res Int. 2011;2011:583139.

4. Piczenik Y, Kjer B, Fledelius HC. Metastatic bacterial endophthalmitis. A report of four cases all leading to blindness Acta Ophthalmol Scand. 1997;75:466-9.
5. Gidley PW, Ghorayeb BY, Stiernberg CM. Contemporary management of deep neck space infections. Otolaryngol Head Neck Surg. 1997;116:16-22.

6. Korman TM, Athan E, Spelman DW. Anaerobic meningitis due to Peptostreptococcus species : case report and review. Clin Infect Dis. 1997;25:1462-4.

7. Brook I. Recovery of anaerobic bacteria from clinical specimens in 12 years at two military hospitals. J Clin Microbiol. 1988;26:1181-8.

8. Mittal MK, Zimmerman RA. Meningitis and epidural abscess related to pansinusitis. Pediatr Emerg Care. 2009;25:267-8.

9. Brook I. Meningitis and shunt infection caused by anaerobic bacteria in children. Pediatr Neurol. 2002;26:99-105.

10. Riggio MP, Lennon A. Specific PCR detection of Peptostreptococcus magnus. J Med Microbiol. 2003;52:309-13.

11. Vallée L, Pinton F, Martin BH, Debray P, Vamecq J, Hladky JP, et al. Brain abscess complicating dental caries in children. Arch Pediatr. 1994;1:166-9.

12. Rosenthal ME, Rojtman AD, Frank E. Finegoldia magna (formerly Peptostreptococcus magnus): an overlooked etiology for toxic shock syndrome?. Med Hypotheses. 2012;79:138-40.

13. Brown MA, Greene JN, Sandin RL, Vincent AL. Case report: anaerobic meningitis caused by Peptostreptococcus magnus after head and neck surgery. Am J Med Sci. 1994;308:184-5.

14. Soga Y, Oka K, Sato M, Kabata T, Kawasaki T, Kawano H, Hayano M. Cavernous sinus thrombophlebitis caused by sphenoid sinusitis - report of autopsy case. Clin Neuropathol. 2001;20:101-5

15. Sato Y, Mizoguchi K, Sato Y, Utsunomiya H, Hayashi T, Kaji M. A case of peptostreptococcal meningitis associated with subarachnoid hemorrhage and subdural hematoma. No To Shinkei. 1986;38:469-73

16. Jiménez Caballero PE, Serviá Candela M. Análisis descriptivo de las meningitis por Streptococcus pneumoniae en un hospital terciario. Neurol Argent. 2012;4:6-10.

17. Luca C, Mihalache D, Luca V, Turcu T. The clinical picture, treatment and prognosis of meningitis due to anaerobic and nonfermentative bacteria. Rev Med Chir Soc Med Nat Iasi. 1999;103:158-60.

18. Walden JH, Hess B, Rigby M. Streptococcal pharyngitis: an uncommon cause of subdural empyema. BMJ Case Rep. $2015 ; 211312$.

19. Giamarellou HJ, Tsagarakis J, Petrikkos G, Mavroudis K, Veldekis D, Daikos GK. Ceftriaxone: therapeutic results in various infections and kinetic studies. Arzneimittelforschung. 1984;34:321-5

20. Warner JF, Perkins RL, Cordero L. Metronidazole therapy of anaerobic bacteremia, meningitis, and brain abscess. Arch Intern Med. 1979;139:167-9.

21. Hendaus MA. Subdural empyema in children. Glob J Health Sci. 2013;5:54-9. 